\title{
Association of atrial fibrillation and left atrial volume index with mortality in patients with COVID-19 pneumonia
}

\section{Antonello D'Andrea ${ }^{1,2 *}$, Vincenzo Russo' ${ }^{2}$, Gianluca Manzo ${ }^{1}$, Valerio Giordano ${ }^{1}$, Marco Di Maio ${ }^{3}$, Fabio Crescibene ${ }^{4}$, Michele D'Alto ${ }^{2}$, and Eduardo Bossone $\mathbb{( i}^{5}$}

\begin{abstract}
${ }^{1}$ Department of Cardiology and intensive coronary unit, "Umberto I" Hospital, Viale S. Francesco 84014 Nocera Inferiore (Salern); ${ }^{2}$ Department of Cardiology, University of Campania "Luigi Vanvitelli", Monaldi Hospital -Via Bianchi - 80100 Naples, Italy; ${ }^{3}$ Division of Cardiology - Maria SS. Addolorata Hospital - Piazza Scuola Medica Salernitana 84025- Eboli (Salern); ${ }^{4}$ Division of Cardiology, M. Scarlato COVID Hospital, Via Passanti 84018 -Scafati (Salern); and ${ }^{5}$ Division of Cardiology, Cardarelli Hospital, Via Cardarelli 80131 Naples
\end{abstract}

Received 18 September 2020; revised 28 October 2020; editorial decision 12 November 2020; accepted 16 November 2020

Coronavirus 2019 disease (COVID-19), caused by SARS-CoV-2, can lead to cardiac impairment. Possible expressions of cardiac injury include increased troponin levels, left ventricular dysfunction, and arrhythmias. ${ }^{1-3}$ Atrial fibrillation (AF) is a common sequela of critical illness, with an estimated prevalence of almost $10 \%$ in Intensive Care Unit (ICU) patients, and several studies report worse outcomes in patients with new-onset $A F$ as compared with their non-AF counterparts. ${ }^{3}$ Information on AF and on left atrial (LA) involvement in patients with COVID-19 pneumonia is limited, and we aimed to explore the possible association with mortality in these patients. This study was conducted from 20 February 2020 to August 2020, in four centres (Umberto I ; M.Scarlato; Cardarelli and Monaldi hospitals); the final date of follow-up was 10 May 2020. All consecutive patients with positive SARS-CoV-2 test result and laboratory- and TCconfirmed interstitial pneumonia were included. Clinical, laboratory, radiological, and ultrasound data were collected. Cardiac injury was defined as blood levels of cardiac biomarkers (high sensitivity Troponin I-ECLIA method; hs-TNI) above the 99th percentile upper reference limit. Left atrial volume was calculated using the biplane area-length method at the apical four-chamber and apical twochamber views at ventricular end-systole (maximum LA size) and indexed for body surface area. Pulmonary artery systolic pressure (PASP) was calculated by adding the value of right atrial pressure to the systolic transtricuspid gradient. Clinical and instrumental variables and outcomes of patients with and without cardiac involvement were compared. The local ethics committee approved the study and all individuals gave written informed consent. A total of 280 hospitalized patients with COVID-19 pneumonia were included in the final analysis; mean age was 66.6years (range, 20-89years), and 112 (40\%) were female. A total of 70 patients had cardiac injury, and these patients were older [mean age 74.5 (38-89) vs. 56.3 (20-79) years; $P<0.001$ ] with increased body mass index [mean 29.3 (25.8$31.4)$ vs. $26.9(22.2-28.3) ; P<0.001]$; had more frequently systemic hypertension [39 (57.5\%) vs. 60 (21.2\%); $P<0.001$ ]; had higher levels of C-reactive protein [mean 99.8 (45.8-130.4) vs. 37.9 (22.2-96.3) $\mathrm{mg} / \mathrm{L} ; P<0.001$ ], and of D-Dimer [mean 4.9 (3.2-7.3) vs. $2.1(0.5-$ 3.4); $P<0.001$ ]; had a higher proportion of multiple ground-glass opacities in computed tomography findings [49 of 70 patients (71.2\%) vs. 72 of 210 patients (34.7\%); $P<0.001]$ and multiple consolidations by lung ultrasound [52 of 70 patients (75.6\%) vs. 77 of 210 patients (36.6\%); $P<0.001]$. By echocardiography, left ventricular (LV) diameters and ejection fraction were comparable between the two groups. Conversely, LV mass index and LA volume index were significantly increased in patients with cardiac injury (Table 1). Greater proportion of patients with cardiac injury showed AF occurrence [28 of $70(40.0 \%)$ vs. 23 of $210(10.4 \%) ; P<0.0001]$ and required invasive or non-invasive mechanical ventilation [45 of 70 (64.2\%) vs. 44 of 210 (21\%); $P<0.001]$. The more common complication in patients with cardiac injury was acute respiratory distress syndrome [47 of $70(67.1 \%)$ vs. 37 of 210 (17.6\%); $P<0.001$ ]. Patients with cardiac injury had higher mortality than those without cardiac injury [34 of $70(48.5 \%)$ vs. 16 of $210(7.6 \%) ; P<0.0001$ ]. In a multivariable Cox proportional hazards model, including clinical data, laboratory measurements, and echocardiographic indexes with potential impact on prognosis as shown by earlier studies, in the overall population of COVID pneumonia patients, troponin levels [hazard ratio (HR), $4.29(95 \% \mathrm{Cl}, 1.85-8.43) P<0.001]$, LA volume index [HR $3.6(95 \% \mathrm{Cl}, 1.15-7.48) ; P<0.001]$, PASP [HR: 3.9; $(95 \% \mathrm{Cl}$, 1.72-6.39) $P<0.001]$, and AF occurrence [HR: $2.5 ;(95 \% \mathrm{Cl}, 1.22-$ 5.4) $P<0.001]$ emerged as independent predictors of in-hospital death. Variance inflation factors were calculated to identify the severity of any multi-collinearity issues in the model, and was lower than 3 for all the considered variables.

Our results showed that higher prevalence of $A F$ in patients with severe COVID-19 pneumonia was associated with higher risk of inhospital mortality. Arrhythmias are complex and multifactorial in a

* Corresponding author. Tel: ++39/0819213377, Email: antonellodandrea@libero.it

Published on behalf of the European Society of Cardiology. All rights reserved. @ The Author(s) 2020. For permissions, please email: journals.permissions@oup.com. 
Table I Echocardiographic structural and systolic/diastolic functional indices in COVID patients with and without cardiac injury

\begin{tabular}{|c|c|c|c|}
\hline Variables & Without cardiac injury $(n=210)$ & With cardiac injury $(n=70)$ & $P$-value \\
\hline Septal wall thickness (mm) & $10.1 \pm 2.3$ & $11.9 \pm 2.3$ & $<0.01$ \\
\hline LV posterior wall thickness $(\mathrm{mm})$ & $9.8 \pm 1.2$ & $10.8 \pm 1.1$ & NS \\
\hline LV end-diastolic diameter (mm) & $48.6 \pm 4.8$ & $49.9 \pm 6.7$ & NS \\
\hline LV end-systolic diameter (mm) & $33.4 \pm 3.8$ & $35.4 \pm 5.2$ & NS \\
\hline $\mathrm{LV}$ mass index $\left(\mathrm{g} / \mathrm{m}^{2}\right)$ & $89.4 \pm 5.8$ & $118.4 \pm 6.8$ & $<0.001$ \\
\hline Mitral peak $E$ velocity $(\mathrm{m} / \mathrm{s})$ & $0.62 \pm 0.32$ & $0.78 \pm 0.3$ & NS \\
\hline Mitral peak $A$ velocity $(\mathrm{m} / \mathrm{s})$ & $0.71 \pm 0.32$ & $0.83 \pm 0.3$ & NS \\
\hline Mitral peak E/A ratio & $0.87 \pm 0.3$ & $0.93 \pm 0.3$ & NS \\
\hline E/Ea ratio & $6.8 \pm 1.3$ & $11.1 \pm 2.1$ & $<0.01$ \\
\hline LV ejection fraction \% & $56.3 \pm 4.8$ & $54.6 \pm 4.7$ & NS \\
\hline $\mathrm{RV}$ basal tract diameter $(\mathrm{mm})$ & 32.22 .6 & 35.84 .2 & $<0.01$ \\
\hline Wall motion score index & $1.05 \pm 0.3$ & $1.23 \pm 0.8$ & $<0.01$ \\
\hline Tricuspid Peak E/A ratio & $0.9 \pm 0.5$ & $0.85 \pm 0.5$ & $<0.05$ \\
\hline $\operatorname{TRV}(\mathrm{m} / \mathrm{s})$ & $2.9 \pm 0.5$ & $3.4 \pm 0.6$ & $<0.001$ \\
\hline PASP $(\mathrm{mmHg})$ & $36.1 \pm 5.3$ & $45.8 \pm 4.8$ & $<0.001$ \\
\hline LA anterior-posterior diameter $(\mathrm{mm})$ & $38.8 \pm 3.5$ & $42.4 \pm 4.6$ & $<0.01$ \\
\hline $\operatorname{LAVI}\left(\mathrm{mL} / \mathrm{m}^{2}\right)$ & $27.2 \pm 8.7$ & $33.9 \pm 4.9$ & $<0.001$ \\
\hline TAPSE $(\mathrm{mm})$ & $20.3 \pm 4.7$ & $16.4 \pm 3.2$ & $<0.01$ \\
\hline
\end{tabular}

LA, left atrium; LAVI, left atrial volume index; LV, left ventricle; PASP, pulmonary artery systolic pressure; RV, right ventricle; TAPSE, tricuspid annular systolic plane excursion; TRV, tricuspid regurgitation velocity.

COVID-19 patient and may result from metabolic derangements, hypoxia, acidosis, intravascular volume imbalances, neurohormonal, and catecholaminergic stress. ${ }^{4,5}$ Sepsis is characterized by a systemic process involving inflammatory cytokines and autonomic dysfunction. Postulated mechanisms of this arrhythmogenesis include autonomic nervous system-induced calcium entry into cardiac myocytes, spontaneous release of calcium from the sarcoplasmic reticulum, ${ }^{6}$ as well as possible direct LA injury due to coronary artery disease with small vessel thrombosis. ${ }^{7}$ Tachycardia and myocardial injury may themselves increase atrial arrhythmias and contribute to worse outcomes. ${ }^{1,2}$

In addition, LA volume index provided independent prognostic information, incremental to clinical data. In our population of patients with higher prevalence of arterial hypertension, and increased both body mass index and LV mass index (independent risk factors for LA thrombosis), ${ }^{8}$ LA volume may reflect the duration and severity of increased LA pressure. In fact, as showed in recent reports, ${ }^{9}$ in adaptation to chronic decreased LV compliance secondary to higher central pulse pressure and LV hypertrophy, LA pressure rises, increasing LA wall tension, and stretching the atrial myocardium. Left atrial stretch and LV pressure overload are the main stimuli for AF occurrence and for secretion of cardiac peptides, levels of which correlated strongly with survival in different clinical settings. ${ }^{10}$

More definitive epidemiologic data are needed. Since some of the therapies empirically used to treat SARS-CoV-2 infections, such as chloroquine, have known effects on myocyte repolarization, resulting in increased risk of QT prolongation and subsequent arrhythmias, and given the high incidence of electrolyte abnormalities in ill patients, ${ }^{1,2}$ high vigilance by the treatment teams is required to avoid iatrogenic harm. Assessment of both LV and LA morphology and function during the recovery of these patients may represent key points in the prognostic stratification.

\section{Acknowledgement}

Conflict of interest: none declared.

\section{References}

1. Shi S, Qin M, Shen B, Cai Y, Liu T, Yang F, Gong W, Liu X, Liang J, Zhao Q, Huang $H$, Yang B, Huang C. Association of cardiac injury with mortality in hospitalized patients with COVID-19 in Wuhan, China. JAMA Cardiol 2020;5: 802

2. Bonow RO, Fonarow GC, O'Gara PT, Yancy CW. Association of coronavirus disease 2019 (COVID-19) with myocardial injury and mortality. JAMA Cardiol 2020;5:751.

3. Goodman S, Weiss Y, Weissman C. Update on cardiac arrhythmias in the ICU. Curr Opin Crit Care 2008;14:549-554.

4. Kuipers S, Klouwenberg PMK, Cremer OL. Incidence, risk factors and outcomes of new-onset atrial fibrillation in patients with sepsis: a systematic review. Crit Care 2014;18:688.

5. Russo V, Di Maio M, Mottola F, Pagnano P, Attena E, Verde N, Di Micco P, Silverio A, Scudiero F, Nunziata L, Fele N, D'Andrea A, Parodi G, Albani S, Scacciatella P, Nigro G, Severino S. Clinical characteristics and prognosis of hospitalized COVID-19 patients with incident sustained tachyarrhythmias: a multicenter observational study. Eur J Clin Invest 2020;e13387.doi: 10.1111/ eci.13387.

6. Otake $H$, Suzuki $H$, Honda T, Maruyama $Y$. Influences of autonomic nervous system on atrial arrhythmogenic substrates and the incidence of atrial fibrillation in diabetic heart. Int Heart J 2009;50:627-641.

7. Mele D, D’Andrea A, Campana M, Di Giannuario G, Flamigni F, Gimelli A, Khoury G, Rizzo M, Moreo A. Non-ischemic ventricular dysfunction in COVID19 patients: characteristics and implications for cardiac imaging on the basis of current evidence]. G Ital Cardiol (Rome) 2020;21:739-749.

8. Zhang X, Hu M, Wang X, Zhang C, Chen W, Chen S, Zhou J, Chen Y, Lou L, Chen G, Dong F, Hu S, Zheng L, Yang J. New perspective on the risk markers 
for left atrial thrombosis in patients with atrial fibrillation. Eur J Prev Cardiol 2020; doi:10.1177/2047487320912084.

9. Przewłocka-Kosmala M, Jasic-Szpak E, Rojek A, Kabaj M, Sharman JE, Kosmala W. Association of central blood pressure with left atrial structural and functional abnormalities in hypertensive patients: implications for atrial fibrillation prevention. Eur J Prev Cardiol 2019;26:1018-1027.

10. Thomas L, Marwick TH, Popescu BA, Donal E, Badano LP. Left atrial structure and function, and left ventricular diastolic dysfunction: JACC state-of-the-art review. J Am Coll Cardiol 2019;73:1961-1977. 